\title{
Do the Renal Function Parameters of Serum and Salivary Urea and Creatinine Alter in Smokeless Tobacco Chewers? A Case- Control Study
}

\author{
Suman Basavarajappa ${ }^{1}$, Shahira $^{2}$
}

\begin{abstract}
Aim: To evaluate and correlate the salivary urea and creatinine levels to the serum levels in smokeless tobacco (SLT) chewers.

Materials and methods: The present study included 60 subjects, 30 SLT chewers, and 30 controls aged between 20 years and 60 years. Serum and salivary urea and creatinine levels were estimated using Berthelot-urease method enzymatic colorimetric method and modified Jaffe's method, respectively.

Results: The mean salivary urea, mean serum, and salivary creatinine levels were higher in SLT chewers $(33.77 \pm 15.04,0.76 \pm 0.17$, and 0.17 $\pm 0.07 \mathrm{mg} / \mathrm{dL}$, respectively) than controls $(32.3 \pm 14.73,0.67 \pm 0.15$, and $0.13 \pm 0.05 \mathrm{mg} / \mathrm{dL}$, respectively). Although serum and salivary urea showed a strongly positive correlation $\left(r=0.654, p<0.001^{* *}\right)$ among SLT chewers and controls, no correlation was noted for serum and salivary creatinine $(r=0.098, p=0.606)$. Receiver operating curve $(\mathrm{ROC})$ analysis revealed better sensitivity and specificity of serum and salivary creatinine than for urea among both SLT chewers and controls.

Conclusion: Salivary urea, serum, and salivary creatinine levels were higher among SLT chewers than controls showing that SLT can be nephrotoxic. Clinical significance: Smokeless tobacco chewers can be assessed for early renal damage caused by the tobacco products using salivary parameters of urea and creatinine so that they can be counseled for the risk of renal diseases and referred appropriately.

Keywords: Creatinine, Saliva, Serum, Smokeless, Tobacco, Tobacco products, Urea.

The Journal of Contemporary Dental Practice (2020): 10.5005/jp-journals-10024-2971
\end{abstract}

\section{INTRODUCTION}

Tobacco use is widespread throughout the world in both smoking and smokeless forms. ${ }^{1}$ Data of the Global Adult Tobacco Survey (GATS-2010) revealed that more than one-third (35\%) of adults in India are tobacco users with $21 \%$ addicted only to smokeless tobacco (SLT) products. This emphasizes the extensive impact that can be rendered by SLT use on human health. Documented evidence of its effects on the cardiovascular system and lungs has now garnered attention on its role in the renal system. Smokeless tobacco contains ingredients such as nicotine, areca nut/betel nut (BN), heavy metals, and various other nephrotoxic agents that are absorbed directly from the oral mucous membrane and can impair renal function and accelerate the progress of renal diseases. ${ }^{2}$

Few studies have reported higher chronic kidney disease (CKD) prevalence in $\mathrm{BN}$ chewers supporting the risk of renal damage due to $\mathrm{BN}$ chewing. Betel nut use is also considered as an important risk factor of CKD in individuals younger than 65 years than are smoking and alcohol. ${ }^{3}$ Proposed mechanism for this includes enhanced synthesis of free radicals by SLT leading to altered glomerular function. Another study in an animal model has shown that the aqueous extract of BN can induce breaks in the DNA of kidney cells. ${ }^{4}$

It is well known that elevated serum urea and creatinine level is associated with abnormal renal function. ${ }^{5}$ Currently, the focus is on employing biofluids, such as, saliva which is a noninvasive, simple, and economical method of assessment of biochemical markers of renal impairment. ${ }^{6}$ A positive correlation between salivary and serum levels of urea and creatinine in patients with moderate and advanced stages of CKD has been recognized. ${ }^{7}$

\footnotetext{
1,2Department of Oral Medicine and Radiology, Government Dental College and Research Institute, Bengaluru, Karnataka, India

Corresponding Author: Suman Basavarajappa, Department of Oral Medicine and Radiology, Government Dental College and Research Institute, Bengaluru, Karnataka, India, Phone: +91 9980839659, e-mail: drsumanag99@yahoo.com

How to cite this article: Basavarajappa S, Shahira. Do the Renal Function Parameters of Serum and Salivary Urea and Creatinine Alter in Smokeless Tobacco Chewers? A Case-Control Study. J Contemp Dent Pract 2020;21(11):1222-1228.

Source of support: Nil

Conflict of interest: None
}

Many studies have considered smoking as a risk for renal disease by analyzing serum urea and creatinine. As there is a paucity of literature regarding these well-recognized renal function parameters in SLT chewers, the present study aimed to evaluate and correlate the serum and salivary urea and creatinine levels in SLT chewers so that any renal impairment if present is detected early and referred appropriately for further management.

\section{Materials and Methods}

This case-control study was carried out in the Department of Oral Medicine and Radiology at Government Dental College and Research Institute, Bengaluru, from September 2018 to 
February 2019. Ethical clearance for the study was obtained from the Institutional ethical clearance committee (GDCRI/IEC-ACM (2)/9/2018-19) following which, a total of 60 subjects (equal number of males and females aged between 20 and 50 years) were recruited for the study.

\section{Inclusion Criteria}

Thirty subjects with a positive history of chewing SLT products for at least 3 years were included as cases and 30 age- and sex-matched subjects without SLT chewing habit/any other deleterious habits were recruited for the study as controls.

\section{Exclusion Criteria}

The study subjects afflicted by any systemic or metabolic disease, taking diuretics, angiotensin-converting enzyme (ACE) inhibitors, immunosuppressants, antidepressants, and chemotherapeutic agents, subjects with various types of cancers, pregnant women, and lactating mothers were excluded from this study. Having obtained voluntary informed written consent from all the study subjects, a detailed case history was recorded in a specially prepared case history pro forma. The study was conducted in accordance with the Helsinki Declaration.

\section{Sample Collection}

Following aseptic conditions, $2 \mathrm{~mL}$ intravenous blood of all the subjects was obtained and centrifuged at 4,000 rpm for 8-10 minutes. The supernatant centrifuged serum was separated. Saliva collection was done between 9.00 a.m. to 12 noon to avoid diurnal variations. Approximately, $3 \mathrm{~mL}$ of saliva was collected using the spitting method, into a sterile graduated tube with the subjects in a seated position for a minimum of 5 minutes and centrifuged at 2,000 rpm for 2-3 minutes. The unstimulated whole saliva sample was immediately subjected to analysis, to avoid deterioration due to incubation and to avoid enzymatic alteration of urea and creatinine in saliva.

Different test tubes were taken and $1 \mathrm{~mL}$ each of the urea and creatinine reagents was pipetted into it. Serum and salivary samples were centrifuged at 1,000 rpm for 10 minutes and the supernatant was obtained. Centrifugation of the serum and saliva samples was done using a centrifuge machine (Remi R-8C BL Laboratory centrifuge) (Fig. 1). A total of $10 \mu \mathrm{L}$ of the supernatant

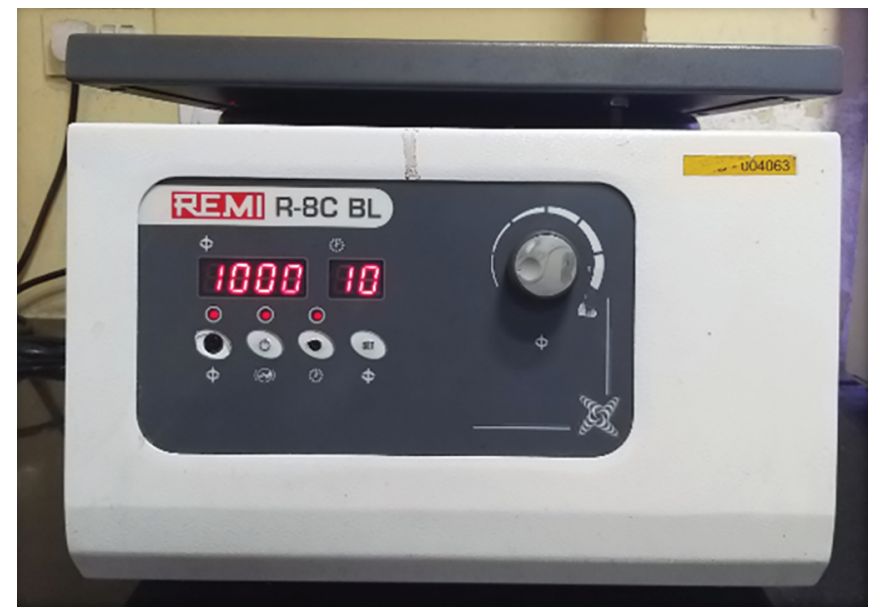

Fig. 1: Centrifuge machine (Remi R-8C BL Laboratory centrifuge)—to centrifuge serum and salivary samples of centrifuged serum and saliva samples were then added to the urea and creatinine reagents. This was then kept in a temperaturecontrolled water bath at $37^{\circ} \mathrm{C}$ for 10 minutes.

\section{Determination of Serum and Salivary Urea Concentration (Berthelot-urease Method Enzymatic Colorimetric Method)}

Urease hydrolyzes urea to ammonia and $\mathrm{CO}_{2}$. A green-colored complex is found when the ammonia formed further reacts with a phenolic chromogen and hypochlorite. The amount of urea present in the sample is directly proportional to the intensity of the color formed. The color change of solution was distinguished and the optical density (OD) was measured at the wavelength of $540 \mathrm{~nm}$ in a photocolorimeter for urea. $^{8}$

$$
\begin{aligned}
& \text { Urea }+\mathrm{H}_{2} \mathrm{O} \stackrel{\text { Urease }}{\rightarrow} 2 \mathrm{NH}_{3}+\mathrm{CO}_{2} \\
& \text { Ammonia + Phenolic chromogen }+ \text { Hypochlorite } \rightarrow \\
& \text { Green-colored complex }
\end{aligned}
$$

\section{Determination of Serum and Salivary Creatinine Concentration (Modified Jaffe's Method)}

Picrate reacts with creatinine to form a red chromophore in the presence of a strong base, such as, $\mathrm{NaOH}$. Based on the principle of enzymatic colorimetry, the rate of increasing absorbance at $510 \mathrm{~nm}$ due to the formation of this chromophore is directly proportional to the creatinine concentration in the sample and was measured using a bichromatic $(510,600 \mathrm{~nm})$ rate technique.

The above analysis was done using an automated biochemistry autoanalyzer (Beckman Coulter AU480) (Fig. 2).

\section{Results}

\section{Statistical Analysis}

All statistical comparisons and graphics were performed with spreadsheet software (Excel, Microsoft). For results on continuous measurements, descriptive and inferential statistical analyses were carried out and the " $p$ " value of $<0.05$ was considered statistically significant.

Analysis of variance (ANOVA), Student's t-test, and Chi-square/ Fisher's exact test were used to find the significance of study parameters. The statistical analysis was carried out by using SPSS

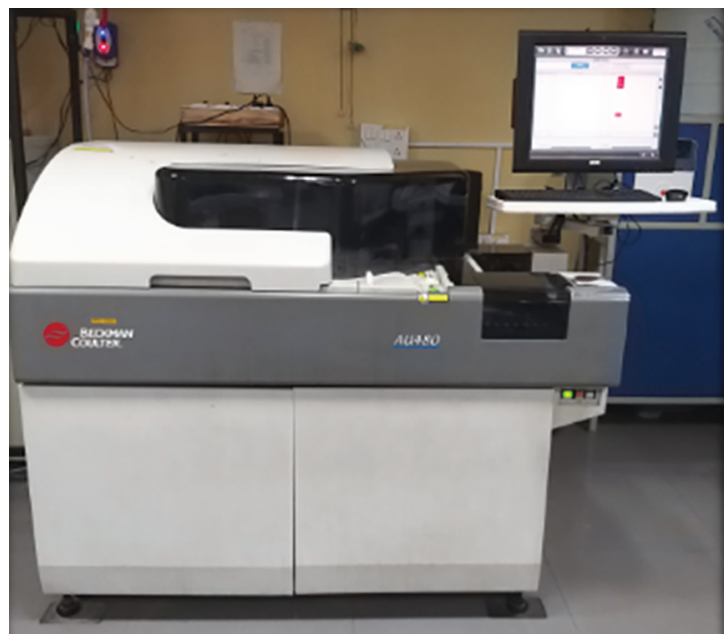

Fig. 2: Automated biochemistry autoanalyzer used for the assessment of urea and creatinine (Beckman Coulter AU480) 
software, version 22. A receiver operating curve ( $R O C)$ was created to find the predictability of study variables for calculating the outcome.

The mean age was $35.53 \pm 9.12$ and $34.80 \pm 8.74$ years for the cases (SLT chewers) and control groups, respectively, with no statistically significant difference $(p=0.752)$. Distribution of frequency and duration of SLT use of the cases (SLT chewers) are shown in Table 1.

The normal concentration of serum urea is $20-40 \mathrm{mg} / \mathrm{dL}$ and of salivary urea is $12-70 \mathrm{mg} / \mathrm{dL}$ and that of serum creatinine is $0.6-1.2$ $\mathrm{mg} / \mathrm{dL}$ and of salivary creatinine is $0.05-0.2 \mathrm{mg} / \mathrm{dL}^{9}$

Among the SLT chewers, mean salivary urea levels (33.77 \pm $15.04 \mathrm{mg} / \mathrm{dL}$ ) were higher while the mean serum urea levels (19.97 $\pm 5.62 \mathrm{mg} / \mathrm{dL}$ ) were lower than in controls, although statistically not significant $(p>0.05)$. Statistically significant higher mean values of serum $(0.76 \pm 0.17 \mathrm{mg} / \mathrm{dL})$ and salivary creatinine $(0.17 \pm 0.07 \mathrm{mg} / \mathrm{dL})$ in SLT chewers were obtained compared with controls $\left(p=0.041^{*}\right.$ and $0.016^{*}$, respectively) (Table 2 ).

The mean serum and salivary urea and creatinine levels showed a linear progressive increase with the increase in the frequency of
SLT chewing habit, although no statistically significant difference was demonstrable. No statistically significant difference was noted between the duration of SLT chewing and the study variables (Tables 3 and 4).

Among SLT chewers, a strong positive correlation $(r=0.654)$ was noted between mean serum and salivary urea levels $(p<0.001)$ with no correlation ( $r=0.098$ ) demonstrable between mean serum and salivary creatinine levels (Table 5). In the control group, a strong positive correlation ( $r=0.759)$ was noted between mean serum and salivary urea levels $(p<0.001)$ and a weak positive correlation $(r=0.375)$ was noted between mean serum and salivary creatinine levels $(p<0.05)$ (Table 5).

The mean serum urea values in female SLT chewers were higher than males $(p=0.032 *)$. No difference was depictable between the genders for salivary urea, serum, and salivary creatinine in either the SLT chewers or controls (Table 6).

The ROC curve for salivary urea showed a sensitivity of 56.67 $(95 \% \mathrm{Cl}=37.4-74.5)$ and specificity of $56.67(95 \% \mathrm{Cl}=37.4-74.5)$ with a positive likelihood ratio (LR) of 1.31 and negative LR of 0.76 .

Table 1: Distribution of frequency and duration of smokeless tobacco use of the cases (SLT chewers)

\begin{tabular}{|c|c|c|c|c|c|c|c|c|}
\hline \multirow{2}{*}{$\begin{array}{l}\text { Cases (SLT } \\
\text { chewers) }\end{array}$} & \multicolumn{4}{|c|}{ Frequency of smokeless tobacco use (times/day) } & \multicolumn{4}{|c|}{ Duration of smokeless tobacco use (in years) } \\
\hline & $1-2$ & $3-5$ & $>5$ & Total & $<6$ & $6-12$ & $>12$ & Total \\
\hline Number & 14 & 13 & 3 & 30 & 17 & 12 & 1 & 30 \\
\hline$\%$ & 46.7 & 43.3 & 10.0 & 100.0 & 56.7 & 40.0 & 3.3 & 100.0 \\
\hline
\end{tabular}

SLT, smokeless tobacco

Table 2: Mean serum and salivary urea and creatinine levels in cases (SLT chewers) and controls

\begin{tabular}{lcccl}
\hline Parameters $(\mathrm{mg} / \mathrm{dL})$ & Cases (SLT chewers) & Controls & Total & p value \\
\hline Serum urea & $19.97 \pm 5.62$ & $20.10 \pm 7.21$ & $20.03 \pm 6.41$ & 0.937 \\
Serum creatinine & $0.76 \pm 0.17$ & $0.67 \pm 0.15$ & $0.71 \pm 0.17$ & $0.041^{*}$ \\
Salivary urea & $33.77 \pm 15.04$ & $32.3 \pm 14.73$ & $33.03 \pm 14.78$ & 0.704 \\
Salivary creatinine & $0.17 \pm 0.07$ & $0.13 \pm 0.05$ & $0.15 \pm 0.06$ & $0.016^{*}$ \\
\hline
\end{tabular}

SLT, smokeless tobacco

${ }^{*} p \leq 0.05$; significant

Table 3: Comparison of mean serum and salivary urea and creatinine levels in cases (SLT chewers) with the frequency of tobacco chewing habit

\begin{tabular}{lccccc}
\hline & \multicolumn{3}{c}{ Frequency of SLT chewing habit (times/day) } & & \\
\cline { 2 - 5 } Parameters $(\mathrm{mg} / \mathrm{dL})$ & $1-2$ & $3-4$ & 5 or more & Total & 0.353 \\
\hline Serum urea & $18.43 \pm 5.29$ & $20.40 \pm 7.23$ & $21.73 \pm 5.26$ & $19.97 \pm 5.62$ & 0.672 \\
Salivary urea & $32.64 \pm 16.20$ & $32.64 \pm 14.51$ & $39.40 \pm 14.62$ & $33.77 \pm 15.04$ & 0.687 \\
Serum creatinine & $0.72 \pm 0.13$ & $0.74 \pm 0.21$ & $0.79 \pm 0.13$ & $0.76 \pm 0.17$ & 0.161 \\
Salivary creatinine & $0.15 \pm 0.05$ & $0.16 \pm 0.05$ & $0.22 \pm 0.11$ & $0.17 \pm 0.07$ & \\
\hline
\end{tabular}

$p>0.05$; not significant

Table 4: Comparison of mean serum and salivary urea and creatinine in cases (SLT chewers) with the duration of tobacco chewing habit

\begin{tabular}{lccccc}
\hline & \multicolumn{3}{c}{ Duration of tobacco chewing habit (in years) } & \multicolumn{1}{c}{ Total } & $p$ value \\
\cline { 2 - 5 } Parameters $(\mathrm{mg} / \mathrm{dL})$ & $<6$ & $6-12$ & $20.00 \pm 0.00$ & $19.97 \pm 5.62$ & 0.316 \\
\hline Serum urea & $18.65 \pm 5.83$ & $22.00 \pm 5.46$ & $40.00 \pm 28.28$ & $33.77 \pm 15.04$ & 0.100 \\
Salivary urea & $28.65 \pm 13.86$ & $40.55 \pm 12.88$ & $0.85 \pm 0.07$ & $0.76 \pm 0.17$ & 0.533 \\
Serum creatinine & $0.73 \pm 0.18$ & $0.78 \pm 0.15$ & $0.15 \pm 0.07$ & $0.17 \pm 0.07$ & 0.907 \\
Salivary creatinine & $0.17 \pm 0.05$ & $0.17 \pm 0.09$ & &
\end{tabular}

$p>0.05$; not significant 
Table 5: Correlation between serum and salivary urea and creatinine between cases (SLT chewers) and controls

\begin{tabular}{llllll}
\hline & \multicolumn{2}{c}{ Cases (SLT chewers) } & & \multicolumn{2}{c}{ Controls } \\
\cline { 2 - 3 } \cline { 5 - 6 } Parameters $(\mathrm{mg} / \mathrm{dL}$ ) & rvalue & $p$ value & & rvalue & pvalue \\
\hline Serum urea vs salivary urea & 0.654 & $<0.001^{* *}$ & & 0.759 & $<0.001^{* *}$ \\
Serum creatinine vs salivary creatinine & 0.098 & 0.606 & & 0.375 & $0.041^{*}$ \\
\hline
\end{tabular}

${ }^{*} p \leq 0.05$; significant

$* * 0<0.001$; highly significant

Table 6: Comparison of mean serum and salivary urea and creatinine levels in cases (SLT chewers) and controls to the gender of the subjects

\begin{tabular}{|c|c|c|c|c|c|}
\hline Parameters (mg/dL) & & Serum urea & Salivary urea & Serum creatinine & Salivary creatinine \\
\hline \multirow[t]{2}{*}{ SLT chewers } & Male & $17.80 \pm 4.09$ & $33.93 \pm 16.02$ & $0.77 \pm 0.18$ & $0.18 \pm 0.08$ \\
\hline & Female & $22.13 \pm 6.22$ & $33.60 \pm 14.55$ & $0.75 \pm 0.16$ & $0.16 \pm 0.05$ \\
\hline Total mean value & & $19.97 \pm 5.62$ & $33.77 \pm 15.04$ & $0.76 \pm 0.17$ & $0.17 \pm 0.07$ \\
\hline$p$ value & & $0.032^{*}$ & 0.953 & 0.750 & 0.410 \\
\hline \multirow[t]{2}{*}{ Controls } & Male & $19.93 \pm 9.23$ & $33.67 \pm 16.49$ & $0.67 \pm 0.19$ & $0.13 \pm 0.05$ \\
\hline & Female & $20.27 \pm 4.74$ & $30.93 \pm 13.17$ & $0.67 \pm 0.11$ & $0.14 \pm 0.05$ \\
\hline Total mean value & & $20.10 \pm 7.21$ & $32.30 \pm 14.73$ & $0.67 \pm 0.15$ & $0.13 \pm 0.05$ \\
\hline$p$ value & & 0.902 & 0.620 & 0.908 & 0.456 \\
\hline
\end{tabular}

${ }^{*} p \leq 0.05 ;$ significant

$p>0.05$; not significant

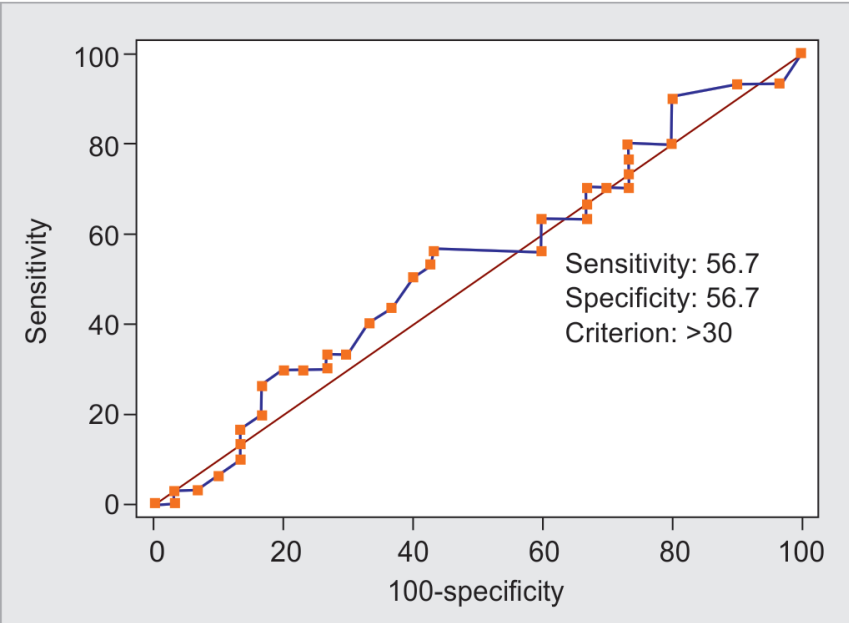

Fig. 3: ROC curve for salivary urea levels. The total area under the curve for salivary urea is 0.53

The area under the curve (AUC) was $0.534(95 \% \mathrm{Cl}=0.401-0.664)$ with no statistically significant difference $(p>0.05)$ (Fig. 3).

The ROC curve for salivary creatinine showed a sensitivity of $63.33(95 \% \mathrm{Cl}=43.9-80.1)$ and specificity of $66.67(95 \% \mathrm{Cl}=$ 47.2-82.7) with a positive LR of 1.90 and negative LR of 0.55 . The AUC was $0.656(95 \% \mathrm{Cl}=0.522-0.774)$ with no statistically significant difference as $p>0.05$ (Fig. 4).

The ROC curve for serum urea showed a sensitivity of 86.67 (95\% Cl $=69.3-96.2)$ and specificity of $23.33(95 \% \mathrm{Cl}=9.9-42.3)$ with a positive LR of 1.13 and negative LR of 0.57 . The AUC was 0.516 (95\% Cl $=0.383-0.647$ ) with no statistically significant difference as $p>0.05$ (Fig. 5).

The ROC curve for serum creatinine showed a sensitivity of 63.33 $(95 \% \mathrm{Cl}=43.9-80.1)$ and specificity of $76.67(95 \% \mathrm{Cl}=57.7-90.1)$ with a positive LR of 2.71 and negative LR of 0.48 . The AUC was 0.647 $(95 \% \mathrm{Cl}=0.513-0.766)$ with no statistically significant difference as $p>0.05$ (Fig. 6).

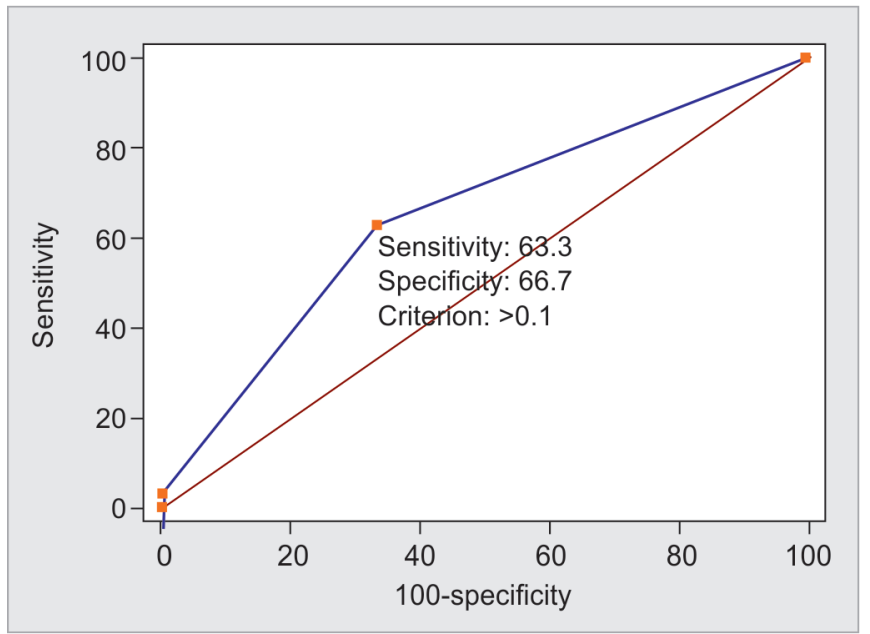

Fig. 4: ROC curve for salivary creatinine levels. The total area under the curve for salivary urea is 0.656

Overall, salivary creatinine (65\%) had the highest accuracy in identifying the renal risk in subjects with SLT use followed by serum creatinine (64\%), salivary urea (53\%), and least by serum urea (51\%). For different values of salivary creatinine, sensitivity and specificity were established and a cut-off value of $>0.1 \mathrm{mg} / \mathrm{dL}$ was determined.

\section{Discussion}

In the current era of alarming trends of tobacco addiction, assessment of its consequence on all the organ systems is inevitable. Studies regarding the effect of chronic tobacco chewing on renal function in subjects without the preexisting renal disease are not extensive. Hence, this study was attempted to evaluate and correlate the levels of serum and salivary urea and creatinine in SLT chewers with healthy controls.

The normal concentration of serum urea is $20-40 \mathrm{mg} / \mathrm{dL}$, of salivary urea is $12-70 \mathrm{mg} / \mathrm{dL}$, of serum creatinine is $0.6-1.2 \mathrm{mg} / \mathrm{dL}$, and of salivary creatinine is $0.05-0.2 \mathrm{mg} / \mathrm{dL} .^{9}$ 


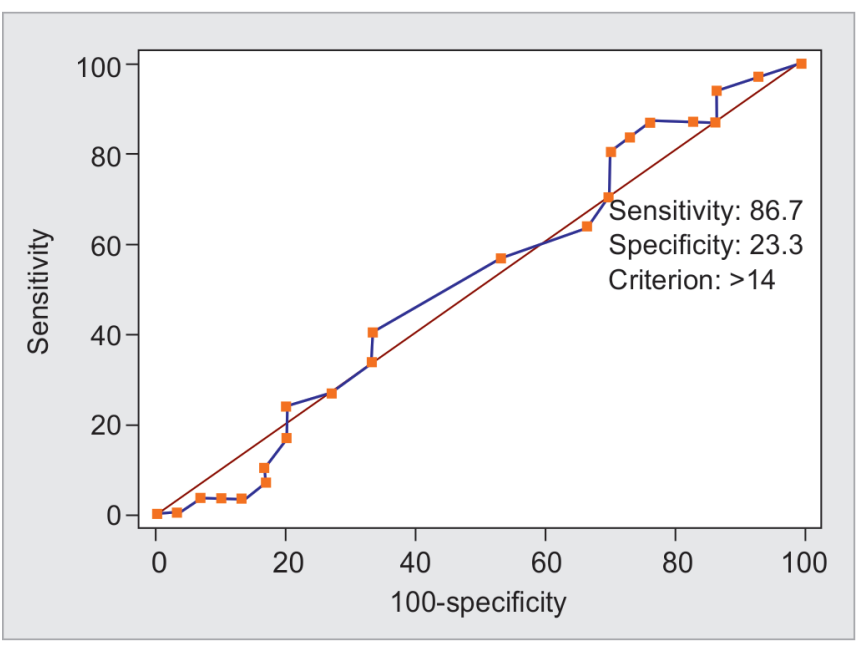

Fig. 5: ROC curve for serum urea levels. The total area under the curve for salivary urea is 0.51

The values obtained in this study were within the normal ranges; yet, subtle changes were noted between SLT chewers and controls. The mean serum urea levels in SLT chewers were lower than controls, although not statistically significant ( $p=0.937$ ) and hence this may not have clinical relevance. This finding is in contradiction to the study by Desai et al. where 100 SLT chewers had higher serum urea levels than controls. ${ }^{5}$ Similar finding was noted by Ahmed et al. in 40 cigarette smokers. ${ }^{2}$ However, mean salivary urea levels in SLT chewers were higher than the controls $(p=0.704)$ though the values were within the normal range. Salivary urea can be produced by oral bacteria (periodontitis) increasing its levels. ${ }^{10,11}$ The poor oral hygiene and periodontitis among SLT chewers and the larger volumes of gingival crevicular fluid generated in periodontitis could have led to such a result in the present study. Increased purine degradation and conversion of hypoxanthine to xanthine by oral bacteria in periodontitis, and thence to urea may also contribute to this. ${ }^{11}$

A statistically significant strong positive correlation $(r=0.654, p$ $<0.001$ ) was noted between serum and salivary urea among cases (SLT chewers) and controls. The above findings agree with the studies by Yajamanam et al., Lasisi et al., Akai et al., and Shannon et al. conducted on CKD patients. .,7,12,13 $^{-13}$

Remaining well within the normal range, the serum creatinine levels in SLT chewers were greater than in control subjects ( $p=$ $0.041^{*}$ ) similar to the findings of other studies in SLT chewers and smokers who also got statistically significant increased levels of serum creatinine. ${ }^{1,2,14}$

Nicotine content and heavy metals in SLT can be nephrotoxic. Smokeless tobacco use can generate higher amounts of free radicals impairing the antioxidant defense system in the kidneys, thereby inducing oxidative stress or lipid peroxidation in the kidneys as noted in an animal study by Al Mukhaini et al. and Avti et al. ${ }^{15,16}$

It has been suggested that creatinine, a waste product of metabolism that is primarily excreted by kidneys, is a very reliable and specific marker for assessment of renal impairment. ${ }^{10}$ Also, serum creatinine has greater prognostic ability compared with urea for predicting the adverse outcomes and progression of kidney disease as creatinine is filtered by the glomerulus. Diminished glomerular filtration rate results in the rise of plasma concentrations of serum creatinine. ${ }^{17}$

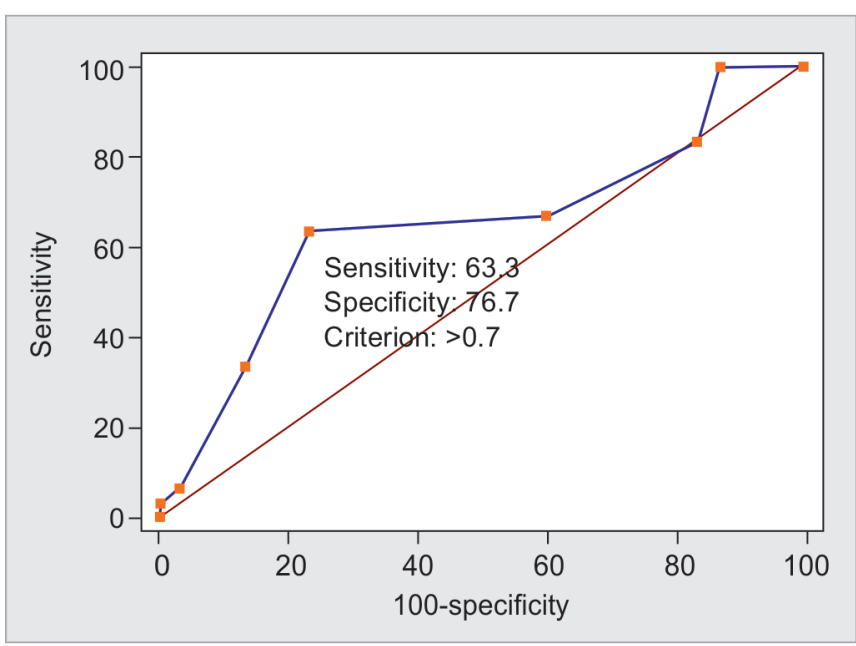

Fig. 6: ROC curve for serum creatinine levels. The total area under the curve for salivary urea is 0.64

Mean salivary creatinine levels in SLT chewers were higher than the controls $\left(p=0.016^{*}\right)$. Creatinine is a large molecule with a high molecular weight that exhibits low lipid solubility, enters saliva from the blood by ultrafiltration. However, due to the relative non-polar nature of creatinine resulting in incomplete filtration, salivary levels are $10-15 \%$ of serum levels. ${ }^{7}$ These findings suggest that salivary creatinine levels can be used to monitor renal function similar to serum creatinine. ${ }^{7,18}$ In healthy individuals, it is unable to diffuse across the cells and the tight junction of the salivary gland, but in patients with increased serum creatinine, a concentration gradient occurs, and creatinine diffusion increases from serum to saliva. ${ }^{19}$ The increase in serum creatinine in patients reflects higher salivary creatinine levels.

Contrary to most of the studies where a positive correlation was established between serum and salivary creatinine in patients with CKD, in the present study no correlation $(r=0.098)$ was noted among the SLT chewers. ${ }^{18,20}$ A weak correlation $(r=0.375)$ was noted between serum creatinine and salivary creatinine among controls with a statistically significant difference between the two as $p<$ 0.05 . Our findings are consistent with previous reports: Lasisi et al., Peng et al., and Venkatapathy et al. conducted on CKD patients ${ }^{6,21,22}$ and are said to be due to the lower serum creatinine levels, with minimal movement of creatinine to saliva due to the lack of a large concentration gradient. Moreover, the geographic variation in the pattern of SLT chewing habits, duration of SLT placement, brands of the SLT, and the amount of nicotine present might have contributed to such findings in our study.

Besides, the mean value of salivary urea in cases and controls was higher than the mean serum urea in cases and controls. This agrees to the study by Dhal Berg et al. and Kovalčíková et al., ${ }^{911,23}$ Unstimulated, slow-flowing saliva may give higher urea values than of serum urea, probably due to contamination by blood, products of bacterial action on food debris or on nitrogenous constituents, other than urea in saliva itself. When the time required for collection is long, such contamination can be greater. In the present study, unstimulated saliva was collected in a time period of 10-15 minutes which supports the above findings. This could be also attributed to the fact that the human parotid saliva has urea concentration which is proportional to or to be below that of blood or plasma urea concentration. This concentration may approach or even surpass 
the blood level at lower flow rates, which is a result of reabsorption of water in the ducts by the salivary glands and active secretion of urea. ${ }^{13}$ The concentration of salivary urea is also influenced by the excretion rate of individual glands. Compared to saliva from the submandibular and sublingual glands, the urea concentration is significantly higher in the parotid gland. ${ }^{24}$ The higher urea concentration in the unstimulated saliva could be further explained based on urea diffusion into saliva in the acini or proximal regions of the salivary ducts, together with sodium and water but not urea reabsorption in the distal regions of the ducts. ${ }^{13}$

A statistically significant higher mean value for serum urea in females than males among SLT chewers was noted in our study. This finding is in accordance with the study by Alsalahi et al. and contrary to the study by Al-Mukhaini et al. ${ }^{15,25}$

Sensitivity and specificity are the basic measures of accuracy of a diagnostic test which depends on how well it separates the group being tested into those with and without the disease in question. Hence, ROC analysis was performed to ascertain the diagnostic potential of serum and salivary urea and creatinine in SLT chewers. The ROC analysis of serum and salivary urea and creatinine in the present study which attempted to ascertain early renal damage in tobacco chewers did not show good accuracy with good sensitivity and specificity. Our finding is not in agreement with those reported by Lasisi et al., Pham et al., and Venkatapathy et al. who found good accuracy with good sensitivity and specificity done in CKD patients with moderate to advanced renal damage. .,22,26 $^{-}$

Accuracy is measured by the area under the ROC curve. The higher AUC (0.65) obtained in our study for salivary creatinine suggests that salivary creatinine is a good alternative diagnostic test to discriminate renal damage in SLT chewers from healthy individuals. Venkatapathy et al. and Xia et al. obtained a large AUC 0.96 and 0.897 , respectively. In the study by Lasisi et al., among CKD patients a cut-off value for salivary creatinine and urea were 0.55 and $27.50 \mathrm{mg} / \mathrm{dL}$, respectively, which gave sensitivity and specificity of 94.0 and $85.0 \%$ for creatinine; as well as 86.0 and $93.0 \%$ for urea. ${ }^{6,22}$ In our study, various cut-off points for serum and salivary urea and creatinine to diagnose renal damage in SLT chewers were obtained using ROC analysis. A cut-off value of $>0.1 \mathrm{mg} / \mathrm{dL}$ gave a sensitivity of $63.3 \%$ and specificity of $66.7 \%$ for salivary creatinine, $>30 \mathrm{mg} / \mathrm{dL}$ with sensitivity of $56.7 \%$ and specificity of $56.7 \%$ for salivary urea, $>0.7 \mathrm{mg} / \mathrm{dL}$ with sensitivity of $63.3 \%$ and specificity of $76.7 \%$ for serum creatinine, and $>14 \mathrm{mg} / \mathrm{dL}$ with sensitivity of $86.7 \%$ and specificity of $23.3 \%$ for salivary urea. That is, above these cut-off values for serum and salivary urea and creatinine the subjects with SLT habits are more likely to develop renal damage and must be subjected to further medical evaluation for appropriate management.

Urea and creatinine are considered as the surrogate markers of renal function and hence do not conclude upon the early renal damage by nephrotoxic agents in SLT products. Tubular enzymes [a- and $\pi$-glutathione S-transferase, $\mathrm{N}$-acetyl-glucosaminidase, alkaline phosphatase, $\gamma$-glutamyl transpeptidase, Ala-(Leu-Gly)aminopeptidase, and fructose-1,6-biphosphatase], low molecular weight urinary proteins $\left(a_{1}\right.$ - and $\beta_{2}$-microglobulin, retinol-binding protein, adenosine deaminase binding protein, and cystatin $\mathrm{C}$ ), $\mathrm{Na}^{+}$/ $\mathrm{H}^{+}$exchanger, neutrophil gelatinase-associated lipocalin, cysteinerich protein 61 , kidney injury molecule 1, urinary interleukins/ adhesion molecules, and markers of glomerular filtration, such as, proatrial natriuretic peptide (1-98) and cystatin C are the early renal biomarkers, detected in urine or serum shortly after tubular injury and contributes to the prediction of early renal impairment. ${ }^{27}$ In addition, salivary cystatin $C$ and BPI fold-containing family A member 2 (BPIFA2), also known as parotid secretory protein are the early renal markers detected in the saliva. ${ }^{28,29}$ However, their analyses are expensive and not widely available.

The present study has some limitations. The amount and type of SLT used should have been evaluated. In tobacco chewers, accurate quantification of the amount of tobacco consumed per unit time (day) is difficult to achieve due to the use of unpackaged SLT and varying contents of net weight of tobacco in different packaged SLT products. Further studies with the larger sample size utilizing a panel of early serological and salivary renal function markers might be helpful to detect the early renal damage caused by the nephrotoxic agents present in SLT or commercial SLT preparations.

\section{Conclusion}

The findings of the present study point to the possible nephrotoxic effect of SLT. Although the well-recognized renal function markers of urea and creatinine are effective in revealing moderate to advanced renal damage, a panel of biochemical glomerular and tubular markers can give a better insight into early renal impairment by tobacco. Smokeless tobacco chewers hence need to be counseled for risk of renal diseases.

\section{Acknowledgments}

The authors would like to express sincere gratitude and appreciation to Dr Vijayalakshmi KR, Head of the Department, Faculty of Oral Medicine and Radiology, GDCRI, Bengaluru, India, for the kind endless help, generous advice, and support during the study.

\section{References}

1. Kumar PS, Jaganmohan P, Rao MS. Assessment of renal damage using selected biochemical markers in smokeless tobacco users. Eur J Biol Sci 2012;4(4):121-125.

2. Ahmed MM, Jawad AS, Osman HM, et al. The effect of smoking cigarette on kidney functions among sundaes peoples. Int J Dev Res 2015;5(5):4473-4475.

3. Kang IM, Chou CY, Tseng YH, et al. Association between betelnut chewing and chronic kidney disease in adults. Int J Occup Med Environ Health 2007;49(7):776-779. DOI: 10.1097/JOM.0b013e318095a48a.

4. Chou CY, Cheng SY, Liu JH, et al. Association between betel-nut chewing and chronic kidney disease in men. Public Health Nutr 2009;12(5):723-727. DOI: 10.1017/S1368980008003339.

5. Desai SV, Nagane NS, Jagtap PE, et al. Tobacco chewing and smokingrisk for renal diseases. Biomed Res 2016;27(3):682-686.

6. Lasisi TJ, Raji YR, Salako BL. Salivary creatinine and urea analysis in patients with chronic kidney disease: a case control study. BMC Nephrol 2016;17(1):1-6. DOI: 10.1186/s12882-016-0222-x.

7. Yajamanam N, Vinapamula KS, Sivakumar V, et al. Utility of saliva as a sample to assess renal function and estimated glomerular filtration rate. Saudi J Kidney Dis Transpl 2016;27(2):312-319. DOI: 10.4103/13192442.178549.

8. Marsh WH, Fingerhut B, Miller H. Automated and manual direct methods for the determination of blood urea. Clin Chem 1965;11(6):624-627. DOI: 10.1093/clinchem/11.6.624.

9. Chand G, Jyoti P, Mahajan M, et al. Correlation of salivary urea and salivary creatinine with blood urea and serum creatinine in patients with chronic kidney disease. Int J Curr Pharm Res 2018;1A(4): 2896-2900. 
10. Hosten AO. BUN and creatinine. Walker HK, Hall WD, Hurst JW, ed. Clinical Methods: The History, Physical, and Laboratory Examinations, ch. 193 3rd ed., Butterworths; 1990.

11. Gaál Kovalčíková A, Pančíková A, Konečná B, et al. Urea and creatinine levels in saliva of patients with and without periodontitis. Eur J Oral Sci 2019;127(5):417-424. DOI: 10.1111/eos.12642.

12. Akai T, Naka K, Yoshikawa C, et al. Salivary urea nitrogen as an index to renal function: a test-strip method. Clin Chem 1983;29(10):1825-1827. DOI: 10.1093/clinchem/29.10.1825.

13. Shannon IL, Feller RP, Eknoyan G, et al. Human parotid saliva urea in renal failure and during dialysis. Arch Oral Biol 1977;22(2):83-86. DOI: 10.1016/0003-9969(77)90082-6.

14. Haider, The effect of smoking cigarette on kidney functions. 2019.

15. Al-Mukhaini N, Ba-Omar T, Eltayeb E, et al. Liver and kidney toxicity induced by Afzal smokeless tobacco product in Oman. Tissue and Cell 2017;49(2):307-314. DOI: 10.1016/j.tice.2017.01.008.

16. Avti PK, Kumar S, Pathak CM, et al. Smokeless tobacco impairs the antioxidant defense in liver, lung, and kidney of rats. Toxicol Sci 2006;89(2):547-553. DOI: 10.1093/toxsci/kfj041.

17. Pandya D, Nagrajappa AK, Ravi KS. Assessment and correlation of urea and creatinine levels in saliva and serum of patients with chronic kidney disease, diabetes and hypertension-a research study. J Clin Diagn Res 2016;10(10):ZC58-ZC62. DOI: 10.7860/ JCDR/2016/20294.8651.

18. Lloyd JE, Broughton A, Selby C. Salivary creatinine assays as a potential screen for renal disease. Ann Clin Biochem 1996;33(5):428-431. DOI: $10.1177 / 000456329603300505$.

19. Renda R. Can salivary creatinine and urea levels be used to diagnose chronic kidney disease in children as accurately as serum creatinine and urea levels? A case-control study. Ren Fail 2017;39(1):452-457. DOI: 10.1080/0886022X.2017.1308256.

20. Temilola DO, Bezuidenhout K, Erasmus RT, et al. Salivary creatinine as a diagnostic tool for evaluating patients with chronic kidney disease. BMC Nephrol 2019;20(1):387. DOI: 10.1186/s12882-019-1546-0.
21. Peng $\mathrm{CH}$, $\mathrm{Xia} Y \mathrm{YC}, \mathrm{Wu} \mathrm{Y}$, et al. Influencing factors for saliva urea and its application in chronic kidney disease. Clin Biochem 2013;46(3):275277. DOI: 10.1016/j.clinbiochem.2012.10.029.

22. Venkatapathy R, Govindarajan V, Oza N, et al. Salivary creatinine estimation as an alternative to serum creatinine in chronic kidney disease patients. Int J Nephrol 2014;2014:742724. DOI: 10.1155/2014/742724.

23. Dhal Berg WH, Sreebny LM, King B. Studies of parotid saliva and blood in haemodialysis patients. J Appl Phys 1967;23(1):100-108. DOI: 10.1152/jappl.1967.23.1.100.

24. Kovalčíková $A$, Janšáková K, Gyuraszova $M$, et al. Salivary creatinine and urea are higher in an experimental model of acute but not chronic renal disease. PLoS ONE 2018;13(7):e0200391. DOI: 10.1371/journal. pone. 0200391.

25. Alsalahi A, Abdulla MA, Al-Mamary M, et al. Toxicological features of Catha edulis (Khat) on livers and kidneys of male and female SpragueDawley rats: a subchronic study. Evid Based Complement Alternat Med 2012;2012:1-11. DOI: 10.1155/2012/829401.

26. Pham TA. Validation of the salivary urea and creatinine tests as screening methods of chronic kidney disease in Vietnamese patients. Acta Odontol Scand 2017;75(8):551-556. DOI: 10.1080/00016357.2017.1356467.

27. Trof RJ, Di Maggio F, Leemreis J, et al. Biomarkers of acute renal injury and renal failure. Shock 2006;26(3):245-253. DOI: 10.1097/01. shk.0000225415.5969694.ce.

28. Alsamarai AT, Husain AA, Saleh TA, et al. Salivary cystatin $C$ as a biochemical marker for chronic renal failure. Eurasian J Anal Chem 2018;13(5):1-8. DOI: 10.29333/ejac/ 95168.

29. Kota SK, Pernicone E, Leaf DE, et al. BPI fold-containing family a member 2/parotid secretory protein is an early biomarker of AKI. J Am Soc Nephrol 2017;28(12):3473-3478. DOI: 10.1681/ASN. 2016121265. 